\title{
Laser-induced phase separation and self-trapping of a laser beam in water-in-oil microemulsions
}

\author{
J.P. DELVILLE, E. FREYSZ, L. SARGER and A. DUCASSE \\ Centre de Physique Moléculaire Optique et Hertzienne, URA CNRS 283, Université Bordeaux I, 33405 \\ Talence cedex, France
}

\begin{abstract}
The thermodiffusive effect induced by a c.w. laser beam in a micellar phase located near a diphasic domain results in a phase separation. The micrometric droplets of the new phase nucleated in the initial one are trapped on the beam axis by electrostrictive effect and self-focuse the laser beam. This self-focusing is characterized by an hysteresis similar to that of the ferromagnetic material magnetization.
\end{abstract}

\section{Résumé}

L'effet thermodiffusif induit par un faisceau laser continu dans une phase micellaire situé près d'un domaine diphasique, conduit à une séparation de phases. Les gouttelettes micrométriques de la nouvelle phase nucléees dans la phase initiale, sont piégées sur l'axe du faisceau par effet électrostrictif et autofocalisent le faisceau laser. Cette autofocalisation est caractérisée par une hystérésis similaire à celle de l'aimantation d'un matériau ferromagnétique. 


\section{Introduction}

In the last few years there was a continuously increasing interest in the interaction between liquid suspensions or liquid mixtures and externally applied fields. Shear flow has been used to induce ordering phenomena in colloïdal dispersions [1]. Uniform electric and magnetic fields applied to electro- and magneto-rheological fluids induce chainlike and columnar structures parallel to the field or liquid to crystal phase transitions [2]. The interaction with a spatially non uniform external applied field has been also studied. Since Ashkin reported on the trapping and the levitation of dielectric suspensions with a strongly focused laser beam [6], radiation pressure effects have been recognized as an important tool for suspended particles manipulation. Particles having a higher refractive index than the surrounding medium will be drawn transversally into high intensity regions. This electrostrictive process explains also the transition observed between a disordered phase and an hexagonal phase in a 2D suspension [3]. Beside this laser induced freezing, it has been recently shown that intense optical fields can induce long range interactions between dielectric particles that can also result in new ordered structures [4].

In this article, we describe a different mechanism which leads to a laser-induced structural phase transition in a mixture. The field modifies locally the concentration of one of the mixture components and generates a phase separation by nucleating droplets of a new phase into the initial one. This inhomogeneity of the medium induces a bistable self-trapped propagation of the laser beam.

\section{Interaction of a laser wave with a microemulsion}

We have analyzed the coupling of the wave with a stable quatemary mixture of water, an oil, a surfactant (soap) and a cosurfactant (alcohol). These mixtures show a very large structural variety. In the poor water (or oil) part of the phase diagram, the mixture has a structure of a micellar phase of microemulsion. It is a stable suspension of very small droplets of water, a few nanometers in diameter (the micelles), isolated from the continuous oil phase by surfactant molecules adsorbed at the water-oil interface. These media are also characterized by phase transitions of liquid-gas type. By varying the temperature, the osmotic pressure or the composition, the initial micellar phase can separate into two micellar phases of different micellar concentrations. In first approximation a micellar phase can be considered as a set of dielectric polarizable spherical particles with a refractive index $n_{w}$ in suspension in a continuous oil phase of index $n_{0}$. If the exciting beam is not a plane wave, it is coupled with the micellar concentration via an electrostrictive process which occurs for the atomic trapping [5] as well as for the laser manipulation of micrometric particles [6]. Electrostriction results from the interaction of the electric field with the dipole induced by the field itself on each particle. It gives rise to a dipolar force proportional to the particle polarizability $\alpha$ and to the intensity gradient which is generally perpendicular to the propagation axis [7]. In the case of water-inoil microemulsions used in this work, the refractive index of the micelles is only sligthly smaller than this of the continuous phase in such a way that the micellar polarisability is weak and negative. The 
electrostrictive moving of the micelles towards the minima of field is then very small and will be neglected in the following.

However, in a liquid mixture, electrostriction is not the only process inducing a variation of micellar concentration. In condensed matter, thermal effects are often important. Even if the wave-medium interaction is not resonant, the field always induces a weak temperature increase due to the absorption of one (or several) component(s) of the mixture. This directly induces a thermal index variation. In our samples this effect is negligible because the medium absorption is of the order of $10^{-4} \mathrm{~cm}^{-1}$ leading to a temperature variation into the beam center approximately equal to $10^{-2} \mathrm{~K}$. Besides the gaussian shape of the beam results in a heating spatially inhomogeneous. The corresponding thermal gradient generates in the mixture a spatial separation of the various components by thermodiffusion. If we consider the micellar phase as a binary mixture of micelles in a continuous phase, thermodiffusion can be taken into account by means of the thermodiffusion ratio $: \mathrm{kT}_{\mathrm{T}}=-\mathrm{T} \operatorname{grad}(\mathrm{c}) / \mathrm{grad}(\mathrm{T})$ where $\mathrm{c}$ is the volumic fraction of micelles and $\mathrm{T}$ the temperature of the system. In micellar phases of microemulsions, $\mathrm{k}_{\mathrm{T}}$ is generally positive and has an important value [8]. Thermodiffusion results then in a statistical moving of the micelles towards the field minima as for electrostriction. So, both processes lead to a decrease of micellar concentration in the center of the laser beam but, in our case, thermodiffusion is dominant.

Such an inhomogeneous variation of micellar concentration generates an inhomogeneous variation of the refractive index of the medium. By analyzing the behaviour of these variations near a critical point of demixtion, it has been previously demonstrated that micellar phases are characterized by giant optical nonlinearities [9]. However these refractive index variations are not the relevant properties for the study reported here. We show instead that the variation of micellar concentration due to the laser manipulation of micelles, can lead to a phase transition into the beam. By taking into account the very small temperature variation $\left(10^{-2} \mathrm{~K}\right)$, this phase transition can be considered as an isothermal process. Along this isotherm, the thermodiffusion which decreases the micellar concentration, induces a local demixtion if the choosen sample is located in the rich micellar part, near the coexistence curve between a micellar phase close to the initial one and a phase of poorer micellar concentration. This phase separation results in the generation inside the laser beam, of droplets constituted by the new phase of weaker micellar concentration in the initial phase. These droplets are characterized by a refractive index larger than this of the initial phase. Their polarisability is positive and the electrostrictive effect due to the field gradient moves them towards the maxima of the beam intensity. We have to notice that the stationary radius of these droplets is of the order of the exciting beam radius (a few micrometers) in such a way that the polarisability of the droplets, which is proportional to their volume, is much larger than this of the micelles whose radius is of the order of few nanometers. Electrostriction can be then negligible on the micelles and important on the droplets. These droplets are trapped in the center of the beam and the medium behaves as a set of self-induced bowl lenses which focuses the laser beam. This self-focusing is quite original since it does not result from a beam propagation in a nonlinear medium. It corresponds instead to a propagation of the laser beam in an inhomogeneous medium that it has induced itself. The figure 1 shows such a propagation. The dark spheres are the droplets trapped in the beam center. Their darkness results from a weaker scattering cross section which corresponds to a droplet composition farther from a critical composition than this of the initial 
phase. The self-focusing in the resulting inhomogeneous medium leads to a propagation into an intense filament also shown in fig. 1.

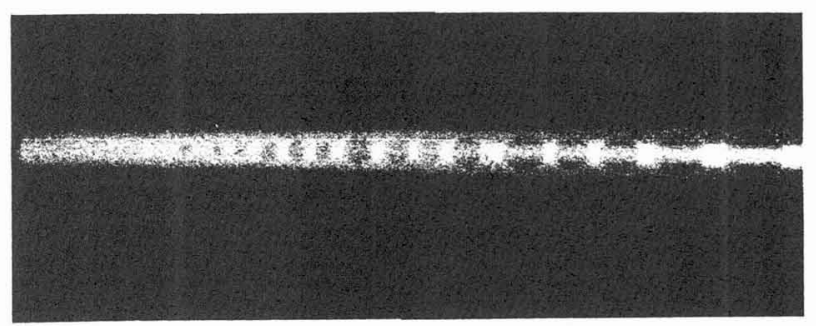

Fig.1 : Self-trapping of the laser beam by the wave-induced droplets of the new phase in the initial one

\section{Experimental results}

All the experiments have been performed with the following quaternary system, in the oil rich domain of the phase diagram : $n$-dodecane (78), water (5), sodium dodecyl sulfate SDS (4.8), n-pentanol (12.2). The mass percentages are indicated in brackets. At room temperature $\left(\mathrm{T}=25^{\circ} \mathrm{C}\right)$, the system features a simple micellar phase. The radius of the micelles essentially depends on the ratio $\mathrm{X}=$ [water] / [surfactant] (1.034). In our case, this mean radius obtained from light scattering or neutron scattering measurements [10] is about $40 \AA$. The very complex phase diagram of the mixture around this composition and this temperature is illustrated in fig. 2. Three different phases $L_{2}, L_{2}^{*}$ and $D$ are observed in the domain. $L_{2}$ is the micellar phase of water micelles in a continuous oil phase previously described. $D$ is a lamellar phase rich in oil, $\mathrm{L}_{2}^{*}$ is a flow birefringent phase [11] also called sponge phase. Fig. 2 presents the evolution of the diagram in the plane $\mathrm{X}=1.034$ for four temperatures : (a) $15^{\circ} \mathrm{C}$, (b) $20^{\circ} \mathrm{C}$, (c) $25^{\circ} \mathrm{C}$, (d) $30^{\circ} \mathrm{C}$, as a function of the mass percentages of $n$-dodecane and n-pentanol [12]. The dotted and hatched domains respectively correspond to the $\mathrm{L}_{2}^{*}$ phase and to a diphasic area in which two micellar phases are in equilibrium. For some particular values of oil and alcohol concentrations the phase separation between the $L_{2}$ phase and the diphasic domain, has a critical behaviour. This results in a critical point $\left(\mathrm{P}_{\mathrm{c}}\right)$ line which is shown in the diagram. The temperature variation of the choosen composition is described by a dotted line in figure 2 .

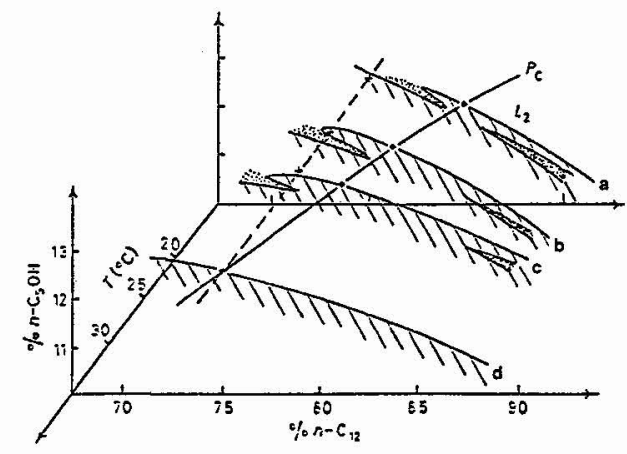

Fig. 2 : Phase diagram of the quaternary system 
This composition crosses the critical line in $\mathrm{T}_{\mathrm{C}}=30^{\circ} \mathrm{C}$ while the low non-critical temperature of phase separation is $\mathrm{T}_{\mathrm{D}}=20^{\circ} \mathrm{C}$. In all the domain $\mathrm{T}_{\mathrm{D}}<\mathrm{T}<\mathrm{T}_{\mathrm{C}}$, it is clearly seen that the sample is located very near the coexistence curve in the one micellar phase region. A small isothermal decrease of the micellar concentration is then sufficient to induce a phase separation.

The mean value of the refractive index of the initial micellar phase is $n_{s}=1.4148$ for a volumic fraction of micelles $\Phi=0.11$. It results from an average between the refractive index of the dispersed phase $n_{d}=$ 1.3914 and this of the continuous phase $n_{c}=1.4174$ [13]. This very small difference $n_{\mathfrak{c}}-n_{d}$ explains that the electrostrictive effect on the micelles is negligible and only efficient on the micrometric poor micellar phase droplets whose refractive index is necessarely included between $n_{S}$ and $n_{\mathcal{C}}$. We have to notice that electrostriction on the micelles can be instead a dominant process for other microemulsions with much larger index contrast between the micelles and the continuous phase [9].

The value of the absorption coefficient, measured by an interferometric method [14] is $\alpha_{\mathrm{a}}=5.5810^{-4} \mathrm{~cm}^{-1}$ and the thermal conductivity is $\Lambda=1.4510^{-3} \mathrm{~W} \mathrm{~cm}^{-1} \mathrm{~K}^{-1}$ [15]. The laser-induced temperature increase can be then calculated [16]. For typical incident laser power of $100 \mathrm{~mW}$ we obtain $\Delta \mathrm{T}=4.410^{-2} \mathrm{~K}$ in the beam center. This very weak increase justifies that the medium evolution can be considered as an isothermal process. It also allows the thermodiffusion induced concentration variation to be evaluated. The thermodiffusion constant $\mathrm{k}_{\mathrm{T}}$ for this sample has not been measured. But, one can assume a $\mathrm{k}_{\mathrm{T}}$ value of the order of 40 at $\mathrm{T}=25^{\circ} \mathrm{C}$ which was measured for another micellar system [8]. We obtain then $\Delta \mathrm{c}=-0.6 \%$ in the beam center. This value is sligthtly larger than the isothermal difference in the phase diagram between the initial phase concentration and this corresponding to the coexistence curve. This sample is then an excellent system to observe a laser-induced phase separation.

A very simple experimental set up presented in figure 3 has been used to quantitatively analyze this selffocusing. The laser beam from on $\mathrm{Ar}^{+}$laser $(\lambda=514.5 \mathrm{~nm})$ is focused in the entrance plane $\pi_{1}$ of the cell (C) containing the microemulsion.

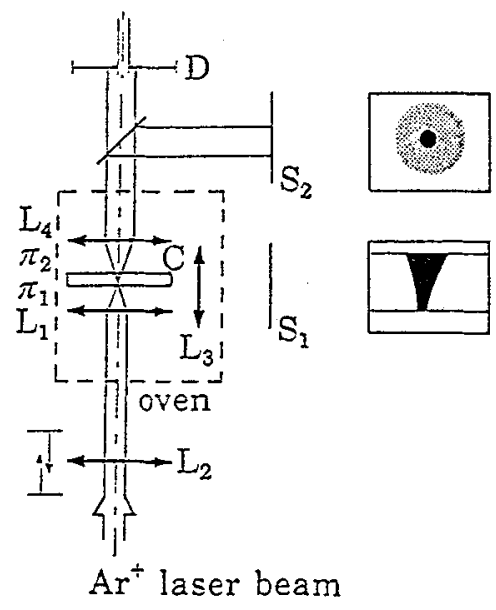

Fig. 3 : Experimental set-up for the analysis of the self-focusing 
The beam-waist $a_{0}$ located in $\pi_{1}$ is varied by changing the optical path between the two lenses $L_{1}$ ( $f=10$ $\mathrm{mm}$ ) and $L_{2}(f=20 \mathrm{~cm})$. Values of $a_{0}$ ranging from 2 to $6 \mathrm{~mm}$ are used for the presented experiments. The lens $L_{3}\left(f=32 \mathrm{~mm}\right.$ ) allows us to check the location of the beam-waist in $\pi_{1}$ and to visualize on the screen $S_{1}$ the propagation of the beam inside the sample. The beam spot in the exit plane $\pi_{2}$ of the cell is imaged by the lens $\mathrm{L}_{4}$ ( $f=32 \mathrm{~mm}$ ) onto a screen $S_{2}$ or in the plane of a circular diaphragm $D$. The diameter of $D$ is chosen so as to transmit only half power of the beam in the absence of nonlinear effects in the medium. The self-focusing of the beam results in an increasing of the $D$ transmission. This is quantitatively analyzed by recording the power transmitted through $\mathrm{D}\left(\mathrm{P}_{\mathrm{V}}\right)$ when increasing the incoming power $\left(\mathrm{P}_{\mathrm{j}}\right)$.

The main results are presented in fig. 4 and fig. 5 [17]. The evolution of the beam transverse profile, observed on $S_{2}$ during a sequence of increasing powers (a) followed by a sequence of decreasing powers (b) is illustrated in fig. 4.

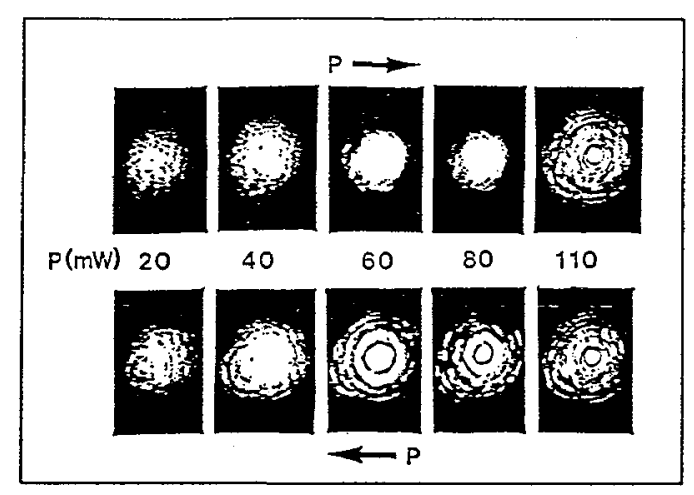

Fig. 4 : Evolution of the beam transverse profile for a sequence of increasing powers and decreasing powers

Figure 5 presents in the first cycle the corresponding variation of $P_{t}$ as a function of $P_{i}$ in a similar sequence. It is very important to note that each profile in fig. 4 or each point of fig. 5 corresponds to a stationary state of the beam propagation in the medium. For each value of $\mathrm{P}_{\mathbf{i}}$, the photograph or the measure of $P_{t}$ is taken after a complete stabilization of the beam propagation. The characteristic time of this evolution depends on the temperature of the sample, but it is always larger than few minutes.

The first sequence of increasing powers in fig. 5 shows that the medium keeps a linear behaviour until a threshold power $\mathrm{P} \uparrow$. The abrupt self-focusing observed at $\mathrm{P} \uparrow$ corresponds to a bifurcation from linear to nonlinear behaviour. The first sequence of decreasing powers clearly demonstrates that the nonlinear properties are preserved when lowering the power below $\mathrm{P} \uparrow$. The linearity is only recovered for powers lower than a second threshold power $\mathrm{P} \downarrow$. 


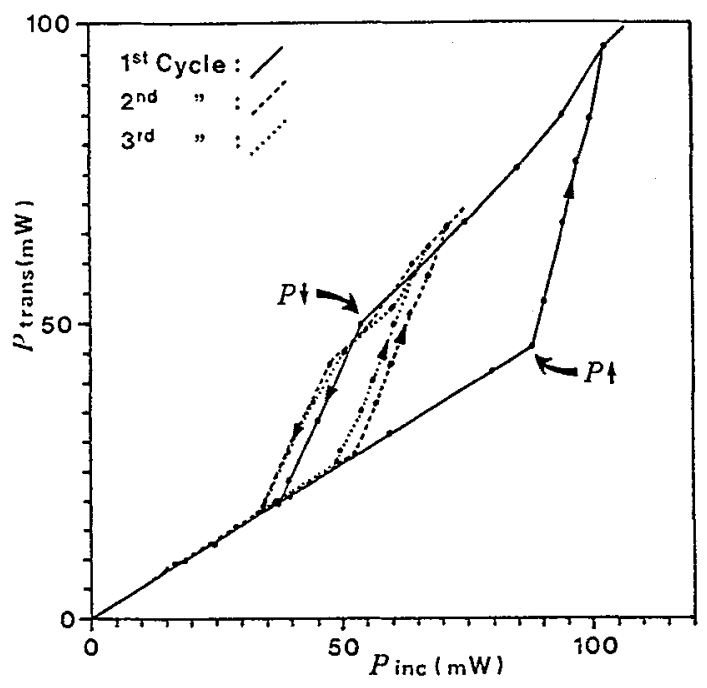

Fig. 5 : Variations of the power $P_{\text {trans }}$ transmitted by the diaphragm $D$ as a function of the incident power $P_{\text {inc }}$ in the non linear medium for three successive sequences of increasing and decreasing powers, $\left(\mathrm{a}_{0}=2.2 \mu \mathrm{m}, \mathrm{T}=24.5^{\circ} \mathrm{C}\right)$.

The 2nd and 3rd experimental cycles presented in fig. 5 show that the medium keeps a memory of its interaction with the wave, when $P_{i}$ is brought back to zero after the first cycle. In these successive runs, performed without waiting between two of them, the value of $\mathrm{P} \uparrow$ decreases and tends quickly to a limit value. At the same time, the value of $\mathrm{P} \downarrow$ varies only weakly. The set of cycles presented in fig. 5 has been obtained during the same day. If waiting until the next day without interaction with the laser, the medium returns to its initial thermodynamical state and the first values of $\mathrm{P} \uparrow$ and $\mathrm{P} \downarrow$ are found again.

\section{Discussion}

Such an hysteresis behaviour has to be interpreted in terms of the dynamics of the laser-induced droplets. We can imagine that it results from the symmetry breaking induced by the field, since the order parameter of such a process could exhibit hysteresis. This interpretation involves a field-induced thermodynamical destabilization of the initial phase in $P \uparrow$ and of the new poor micellar phase in $P \downarrow$. The stability analysis of the rising and the lowering branches of the first cycle shows a more complicated behaviour. If in $\mathrm{P} \uparrow$ the expected behaviour is observed, it is different in $\mathrm{P} \downarrow$ : a small increase of the incident power below $\mathrm{P} \downarrow$ induces a smooth increase of the beam self-focusing. The return to a linear propagation in $\mathrm{P} \downarrow$ does not result then from a thermodynamical destabilization of the droplets and a more advanced interpretation has to be found. First, in order to characterize more precisely the destabilization mechanism in $\mathrm{P} \uparrow$ we have analysed the temperature variation of this threshold power. The experimental results shows that $\mathrm{P} \uparrow$ diverges when approaching the critical temperature with a critical exponent equal to 0.28 . This, surprising behaviour is in contradiction with our initial interpretation. Indeed, if $\mathrm{P} \uparrow$ was just controlled by the droplet 
nucleation process, it would vanish in the vicinity of the critical point because of the diverging behaviour of the osmotic compressibility in this point. So the beam self-focusing is not only induced by the nucleation process. In fact, this original self-focusing is generated by the bowl lens behaviour of the trapped droplets whose index contrast results from the contrast of micellar fraction between the two phases. Since this contrast vanishes when approaching the critical point, the self-focusing threshold $\mathrm{P} \uparrow$ must diverge at this point.

This discussion shows that two contributions are needed in order to induce the wave self-focusing. It is necessary to induce first the phase transition. In our experimental case, it is realized by thermodiffusion. The corresponding threshold power $(\mathrm{P} \uparrow)_{\mathrm{th}}$ is inversely proportional to the thermodiffusion ratio $\mathrm{kT}$. Afterwards these nucleated droplets have to be trapped on the beam axis in order to focuse the laser beam. This trapping results from an electrostrictive process. The corresponding threshold power $(\mathrm{P} \uparrow)_{\mathrm{rad}}$ is inversely proportional to the index contrast between the new poor micellar concentration phase and the initial one. This index contrast can be estimated by using the Clausius-Mossoti relation. A simple calculation shows that it behaves like the micellar fraction contrast which vanishes near the critical point with the critical exponent $\beta(\beta=0.31)$. Moreover $\mathrm{k}_{\mathrm{T}}$ is known to behave near the critical point like the correlation length of the fluctuations $\xi$ [18]. Then it diverges with the critical exponent $v(v=0.63)$. It results that $(\mathrm{P} \uparrow)_{\text {th }}$ vanishes at the critical point whereas $(\mathrm{P} \uparrow)_{\mathrm{rad}}$ diverges. Accordingly, the laser beam selffocusing, which needs both nucleation and trapping, is characterized by a threshold power $\mathrm{P} \uparrow$ determined by the larger of the two thresholds $(\mathrm{P} \uparrow)_{\mathrm{th}}$ and $(\mathrm{P} \uparrow)_{\mathrm{rad}}$.

Far enough from the critical temperature $(\mathrm{P} \uparrow)_{t h}$ is larger than $(\mathrm{P} \uparrow)_{\mathrm{rad}}$. The laser beam self-focusing is controlled by droplet nucleation. When the incident power reaches $\mathrm{P} \uparrow$ (which corresponds in this case to $\left.(\mathrm{P} \uparrow)_{\mathrm{tb}}\right)$ a first droplet is nucleated at the entrance of the cell containing the sample. This droplet is automatically trapped by the beam $\left((\mathrm{P} \uparrow)_{\mathbf{r a d}}<(\mathrm{P} \uparrow)_{\mathbf{L}}\right)$ and behaves like a bowl lens. At the focal point of this induced lens, the nucleation conditions are satisfied again. It results that a second droplet is generated which focuses light and so on. This destabilization process in $\mathrm{P} \uparrow$ gives rise to the strong laser beam selffocusing shown in the first cycle of fig. 5. When decreasing now the incident power, no more droplets are nucleated at the entrance face of the cell. However, the strong focusing resulting from the droplets in the beam implies that the nucleation conditions remain satisfied at all the intermediate beam waists generated by these droplets. This irreversible nucleation process leads to the optical hysteresis illustrated in fig. 5 .

Close enough from the critical point, the trapping threshold $(\mathrm{P} \uparrow)_{\text {rad }}$ is larger than the nucleation threshold $(\mathrm{P} \uparrow)_{\text {th. }}$. The laser beam propagation is then completely dominated by the droplet trapping and the lens effect. In this case, the self-focusing threshold $\mathrm{P} \uparrow$ corresponds to $(\mathrm{P} \uparrow)_{\mathrm{rad}}$. As we have seen before it must diverge at the critical point vicinity with the exponent $\beta$. The measured exponent 0.28 is in good agreement with this predicted behaviour. In this case, the hysteresis interpretation is a little more complicated. When the incident power reaches $(\mathrm{P} \uparrow)_{\mathbf{t h}}$ a first droplet is nucleated. However its index contrast is too small to generate its trapping at the beam center. No self-focusing is expected. In order to obtain this behaviour, an increase of the incident power is needed. Then more and more droplets are nucleated and when $(\mathrm{P} \uparrow)_{\mathrm{rad}}$ is reached their index contrast becomes sufficient for their trapping. It results that in ( $\mathrm{P} \uparrow)_{\mathrm{rad}}$ the field traps a large number of droplets which generate a strong self-focusing. By reducing the incident power we observe again an optical hysteresis behaviours. It can be explained by the fact that the thermodiffusive process 
remains efficient on these trapped droplets. We have seen that their stationary radius is equal to the beam radius. So, it exists a thermal gradient inside these droplets wich induces a micelle ejection by thermodiffusion. This process increases the index contrast. It induces an irreversible increase of the trapping and of the lens effects in spite of the incident power decreasing. This irreversible evolution of the index contrast is responsible of the observed optical hysteresis behaviour. Moreover we must notice that this thermodiffusive effect inside the droplets is also efficient for a self-focusing threshold controlled by nucleation $\left((\mathrm{P} \uparrow)_{\operatorname{rad}}<(\mathrm{P} \uparrow)_{\mathrm{th}}\right)$, but it is not the prevailing process in this case.

We have experimentally observed these two cases of abrupt self-focusing as well as the intermediate case $\left((\mathrm{P} \uparrow)_{\mathrm{th}} \approx(\mathrm{P} \uparrow)_{\mathrm{rad}}\right)$ in which the laser beam self-focusing does not present an unstable character and is more progressive. This loss of real threshold is related to the aberrations induced on the wave by the droplets which can no longer be neglected when the two threshold powers are close together. The aberrations result from the fact that droplets and beam radii are of the same order. The nucleation conditions and the trapping are disturbed at the focal point associated to a first droplet. Thus the instability mechanism responsible of the strong self-focusing is disturbed too.

All these different optical hysteresis behaviours are, in some respects, very similar to the hysteresis associated to a ferromagnetic material magnetization by a magnetic field. Indeed, as we have seen before, the bistable behaviours of the beam propagation results from three contributions : the thermodynamical destabilization of the initial phase characterized by droplet nucleation at a threshold $(\mathrm{P} \uparrow)_{\text {th }}$, the trapping of these droplets at the threshold $(\mathrm{P} \uparrow)_{\mathrm{rad}}$ and the irreversible modifications of the medium : nucleation at different intermediate beam waists and increase of the index contrast by thermodiffusion inside the droplets. The ferromagnetic hysteresis is qualitatively explained by analogous arguments : nucleation field, propagation field and irreversible modifications of the medium. When one of these two fields dominates the other we obtain a square hysteresis cycle. If these two fields are of the same order, the hysteresis cycle is less steep. In order to show more precisely this analogy of behaviours, we have performed typical experiments similar to those of macroscopic ferromagnetism. For example, it is well known that a small number of increasing-decreasing magnetic field variations have to be made before obtaining a stationary hysteresis cycle [19]. The second and the third experimental cycles presented in fig.5 illustrate a similar behaviour.

The medium keeps a memory of its interaction with the wave and a limit cycle is obtained from the third cycle. Before analysing this memory effect, the behaviour of $\mathrm{P} \downarrow$ must be described. The transition between the nonlinear to the linear propagation results from two different processes. First the droplet formation is stopped since nucleation conditions are no longer satisfied at intermediate beam waists. Then, the droplets progressively leave the beam because the trapping effect is less and less efficient compared to the thermal and hydrodynamical fluctuations when decreasing the incident power. Out of the laser beam these just untrapped droplets are characterized by a strong metastability. Their size slowly decreases. Moreover their large radius (a few micrometers) induces a very small mass diffusion constant. After an hour the diffusion length varies from five to fifteen micrometers for a droplet radius variing from $a_{0}$ to $a_{0} / 10$ where $a_{0}=5 \mu \mathrm{m}$ is the beam waist. Next these untrapped droplets behave as nucleation centers if we perform a second cycle consecutively to the first one. The presence of these nuclei in the medium induces a lowering of the nucleation barrier and leads to a reduction of the phase transition threshold $(\mathrm{P} \uparrow)_{\mathrm{L}}$. Moreover the 
thermodiffusion inside the droplets when they were trapped in the beam involves a large index contrast of these nuclei. This increase of the index contrast between a just nucleated droplets at $\mathrm{P} \uparrow$ and a droplet

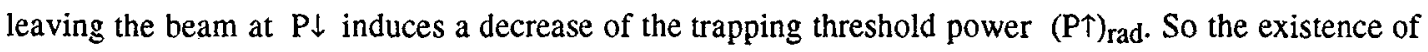
these large index contrast nuclei explains the decrease of $\mathrm{P} \uparrow$ from cycle to cycle, illustrated in fig. 5 . It also explains the small decrease of $\mathrm{P} \downarrow$. Indeed, this variation of the index contrast increases the trapping and the lens effects and delays the stopping of the droplet nucleation and the loss of trapping. It results that $\mathrm{P} \downarrow$ decreases from a cycle to another. Moreover, this variation of the threshold powers is characterized by limit values because the nucleus size and the refraction index are obviously smaller than the size of the exciting field and the refraction index of the oil continuous phase.

The analogy between our optical hysteresis and the ferromagnetical hysteresis can be detailed by analysing the cycle creep phenomenon [20]. If a ferromagnetical material is submitted to dissymmetric magnetic field increasing-decreasing variations, the hysteresis cycles move slowly towards increasing magnetization. This phenomenon results from the combination of three properties of the medium : the existence of long range coupling inside the domain structure, the existence of multiple domain configurations of close macroscopic energies and the possibility for the system to go from one to another at random. In submitting our sample to dissymmetric incident power increasing-decreasing variations we observed a laser beam self-focusing of increasing quality. In our system, long range couplings between the droplets are provided by the small droplet sedimentation in the laser beam resulting from the small density contrast of these droplets [21]. These irreversible hydrodynamical couplings result from the long range perturbation of the flow around a moving sphere at low Reynolds number. Moreover nucleation processes are analogous in magnetism and phase transition providing by this way the two last conditions required for cycle creep. So, dissymmetric incident power increasing-decreasing variations generates a more and more structured and contrasted medium which leads to a laser beam self-focusing of increasing quality.

These first experimental results demonstrate that a laser wave is an interesting tool to control structural changes in liquid mixtures via variations of component concentrations. The study of the spinodal decomposition, in particular, is now in progress in our group using an induced grating method.

\section{References}

[1] Ackerson B.J., Physica A174 (1991) 15.

[2] Hasley T.C., Toor W., Phys. Rev. Lett. 65 (1990) 2820.

Tao R., Woestman J.T., Jaggi N.K., Appl. Phys. Lett. 55 (1989) 1844.

Lemaire E., Grasseli Y., Bossis G., J. Phys. II France 2 (1992) 359.

[3] Chowdhury A., Ackerson B.J., Clark N.A., Phys. Rev. lett. 55 (1985) 833.

[4] Burns M.M., Fournier J.M., Golovchenko J.A., Science 249 (1990) 749.

[5] Phillips W.D., Metcolf H., Phys. Rev. Lett. 48 (1982) 596.

[6] Ashkin A., Phys. Rev. Lett. 24 (1970) 156.

[7] Palmer A.J., Opt. Lett. 5 (1980) 54.

[8] Jean-Jean B., Freysz E., Ducasse A., Pouligny B., Europhys. Lett. 7 (1988) 219.

[9] Jean-Jean B., Freysz E., Ponton A., Ducasse A., Pouligny B., Phys. Rev. A39 (1989) 5268. 
[10] Bellocq A.M., Roux D., in "Microemulsions : Structure and Dynamics". S. Freiberg and P. Bothorel ed. (Chemical Rubber, Boca Raton, FL, 1987).

[11] Cates M.E., Roux D., Andelman D., Milner S.T., Safran S.A., Europhys. Lett. 5 (1988) 733.

[12] Gazeau D., Freysz E., Bellocq A.M., Europhys. Lett. 9 (1989) 883.

[13] Rebbouch N., Lalanne J.R., J. Chem. Phys. 90 (1989) 1175.

[14] Canioni L., Sarger L., Segonds P., Private communication.

[15] Dorion P., Lalanne J.R., Pouligny B., Imaizumi S., Garland C.W., J. Chem. Phys. 87 (1987) 578.

[16] Gordon J.P., Leite C.C., Moore R.S., Porto S.P.S., Whinnery J.M., J. Appl. Phys. 36 (1965) 3.

[17] Ponton A., Delville J.P., Freysz E., Ducasse A., Bellocq A.M., Europhys. Lett. 17 (1992) 27.

[18] Giglio M., Vendramini A., Phys. Rev. Lett. 34 (1975) 561.

[19] Porteseil J.L., Phys. Lett. 121 A (1987) 145.

[20] Néel L., J. Phys. Rad. 20 (1959) 215.

[21] Hinch E.J., "Sedimentations of Small Particles" in Disorder and Mixing, E. Guyon et al. ed., Nato ASI 152 (1988) 153. 